\title{
Molecular characterization of hemagglutinin-neuraminidase fragment gene of Newcastle disease virus isolated from periodically-vaccinated farms
}

\author{
Lucia S. Triosanti ${ }^{1}$, Michael Haryadi Wibowo ${ }^{1}$ and Rini Widayanti ${ }^{2}$
}

\author{
1. Department of Microbiology, Faculty of Veterinary Medicine, Gadjah Mada University, Yogyakarta, \\ Indonesia; 2. Department of Biochemistry, Faculty of Veterinary Medicine, Gadjah Mada University, \\ Yogyakarta, Indonesia. \\ Corresponding author: Michael Haryadi Wibowo, e-mail: mhwibowo@ugm.ac.id \\ Co-authors: LST: Is.triosanti@gmail.com,RW: rini_widayanti@ugm.ac.id \\ Received: 20-12-2017, Accepted: 19-04-2018, Published online: 20-05-2018
}

doi: 10.14202/vetworld.2018.657-666 How to cite this article: Triosanti LS, Wibowo MH, Widayanti R (2018) Molecular characterization of hemagglutinin-neuraminidase fragment gene of Newcastle disease virus isolated from periodically vaccinated farms, Veterinary World, 11(5): 657-666.

\begin{abstract}
Background and Aim: Newcastle disease (ND) caused by avian paramyxovirus serotype-1 (APMV-1) is long known as an acute contagious and infectious disease of various bird species. Prior studies have acknowledged that the virus could cause up to $100 \%$ morbidity and mortality as well as reducing eggs production. In theory, hemagglutinin-neuraminidase (HN) in ND virus (NDV) is one of the surface glycoproteins that functions during the attachment, assembly, and maturation of the virus. On the fields, Indonesia has been recognized as an endemic country for ND where continuous outbreaks of ND in commercial chicken farms have been reported despite the implementation of periodical vaccination programs. Thus, this study aims at characterizing NDV isolated from periodically vaccinated commercial farms, comparing its genetic correlation based on their HN gene fragment with registered NDV originated from Indonesia as well as with existing vaccine strains.
\end{abstract}

Materials and Methods: The HN gene fragment of NDV isolated from well-vaccinated farms was amplified using primer pairs of forward 5' GTGAGTGCAACCCCTTTAGGTTGT 3' and reverse 3' TAGACCCCAGTGATGCATGAGTTG 3' with a 694 bp product length. The nucleotide sequences of nine samples, which were gathered from Kulon Progo, Gunung Kidul (2), Boyolali (2), Magelang, Muntilan (2), Palembang, and Medan, were later compared with the sequences of HN gene of NDV available in NCBI Genbank database. The amino acid sequence analysis and multiple sequence alignment were conducted using the Mega7 program.

Results: The data analysis on amino acid sequences showed that the structure of amino acid residue at positions 345-353 for all isolates appears to be PDEQDYQIR. The structure is the same as for archived samples from Indonesia and either LaSota or B1 vaccine strains. The amino acid distance between observed isolates and LaSota vaccine strain is $8.2-8.8 \%$ with a homology value at 91.2-91.7\%.

Conclusion: Looking at amino acid sequence analysis, LaSota vaccines can considerably be stated as being protective against ND disease outbreak. However, the distant homology value from a perfect condition for the protection might have acted as the root cause of vaccination failures.

Keywords: hemagglutinin-neuraminidase, Newcastle disease, protein, reverse transcriptase polymerase chain reaction, sequencing, vaccination, virus.

\section{Introduction}

Newcastle disease (ND; known as tetelo disease in Indonesia) has been reported as critically affecting the economics of poultry industry [1]. In fact, the virus has caused numerous economic losses in the industry including morbidity and mortality rates that may reach up to $100 \%$ along with decreased egg productions $[1,2]$. In the world, the ND virus (NDV) worldwide spread and high transmission have even made the disease to get included in the LISTED (reportable disease) by World Organization for Animal

Copyright: Triosanti, et al. Open Access. This article is distributed under the terms of the Creative Commons Attribution 4.0 International License (http://creativecommons.org/licenses/ by/4.0/), which permits unrestricted use, distribution, and reproduction in any medium, provided you give appropriate credit to the original author(s) and the source, provide a link to the Creative Commons license, and indicate if changes were made. The Creative Commons Public Domain Dedication waiver (http:// creativecommons.org/publicdomain/zero/1.0/) applies to the data made available in this article, unless otherwise stated.
Health [3]. At the national level, ND has been reported in Indonesia as an endemic disease because its cases have been reported throughout the year in various regions [4].

In Indonesia, an intensive vaccination in conjunction with good management of chicken farms has been considered as a comprehensive strategy to prevent outbreaks of ND $[5,6]$. In particular, various vaccination programs and commercial type vaccines (either living or killed vaccines) have been applied in Indonesia. In the field practice, vaccinations are conducted either independently for a single disease (ND) or in combination to prevent a set of different poultry diseases [7].

Despite a continuous implementation of the vaccination programs, ND still stands as a major problem in the poultry industry, in which numerous cases of ND are continuously reported in periodically vaccinated chicken farms. Despite the critical importance 
of hemagglutinin-neuraminidase $(\mathrm{HN})$ as a surface glycoprotein during the attachment, assembly and maturation of NDV, however, there is no report on the characterization of HN in NDV gene isolated from Indonesia. Therefore, this study first aims at characterizing outbreak-causing NDV based on the HN gene fragment. To investigate the cause of continuous occurring of ND despite a consistent implementation of vaccination programs, sample viruses need to be isolated from well-vaccinated commercial poultry farms. The results will be the first scientific report on the characteristics of $\mathrm{HN}$ gene isolate virus from Indonesia.

Then, this study was proposed to observe any genetic correlation between observed samples isolated in this study, existing isolated samples from Indonesia that have been previously recorded at GenBank database and the strain of LaSota vaccine.

\section{Literature Review}

The NDV was first recorded to cause an outbreak in 1926 in Java Island, Indonesia [8], and in 1927 near Newcastle-upon-Tyne, England [9]. Years later, ND subsequently spread and became endemic in many countries [2,10]. In Asia, Africa and Central as well as South America, the epizootics of ND in poultry continued to occur, while sporadic epizootics occurred in Europe [11,12]. During 2009-2010, there was a report of ND outbreaks that occurred at various commercial chicken farms in Indonesia, resulting in an around $80 \%$ mortality rate [13]. The morbidity and mortality rates in different flocks were in fact varied, following the variation of respective NDV strains. According to Darminto and Ronohardjo [14], losses that might considerably be related to the continuous occurrences of ND disease in Indonesia have been estimated to reach beyond US\$10 million annually.

In the literature, ND disease has been noted to be caused by avian paramyxovirus serotype-1 (APMV1), which belongs to family Paramyxoviridae, genus Avulavirus, Avian paramyxovirus serogroup [15]. In details, the virus has a non-segmented, negative sense, and single-stranded RNA genome [16,17]. Regarding its size, the NDV genome has an approximately $15.2 \mathrm{~kb}$ in length $[18,19]$. Regarding its content, the virus genome encodes six structural proteins including nucleoprotein $(\mathrm{NP})$, phosphoprotein $(\mathrm{P})$, matrix $(\mathrm{M})$, fusion protein $(\mathrm{F}), \mathrm{HN}$ and dependent RNA polymerase (L). Besides, there are two non-structural proteins (V and $\mathrm{W}$ proteins) produced during the transcription of $\mathrm{P}$ gene. $\mathrm{HN}$ and $\mathrm{F}$ proteins are located on the virus membrane surface, while N, P and L proteins form the genetic material complex of the virus. Practically, NDV proteins deliver the virulence within a host's body [20]. First, an NDV infection is initiated by a receptor recognition and the virion binding to sialoglycoconjugates on the host cell surface. The binding process is followed by a fusion of the viral lipid envelope with cell host membrane [21]. Then, the interaction of two viral surface glycoproteins, i.e. HN and F, accomplishes the NDV infection process [22].

In particular, $\mathrm{HN}$ protein has been recognized as having a critical contribution during NDV pathogenesis $[23,24]$. The protein delivers their multifunction in the attachment process between virus and host. In the release of virus from an infected cell, it produces neuraminidase (NA) activity [21]. During the attachment process of NDV to a host cell, HN protein acts as an agent to recognize a sialic acid receptor on the surface of targeted host cells. Besides, the protein triggers a fusion process of viruses in target cell membranes in conjunction with $\mathrm{F}$ protein. Theoretically, $\mathrm{F}$ protein requires cooperation with $\mathrm{HN}$ protein to perform a membrane fusion during the fusion process so that the virus may penetrate a host cell's surface $[25,26]$.

Furthermore, HN protein has discovered to vary regarding its length. In details, nine different variants have been reported, i.e. 570 amino acids (570aa), 571 aa, 572aa, 577aa, 578aa, 586aa, 582aa, and 616aa $[19,27]$. The length of HN protein, however, does not affect the virulence of NDV [28]. Within itself, HN protein has five antigenic sites, i.e. amino acid residues 193-201, 345-353, 494, 513521 , and 569 [29]. In theory, HN protein has been known to have a critical role in inducing immunity against different viral infections. However, the protein is susceptible to the immune defense system of an infected individual host. The vulnerability during an encounter then makes the protein to simultaneously response by straightforwardly producing antigenic variations $[29,30]$. In particular, the amino acid residue at position 347 in $\mathrm{HN}$ protein is critical for virus recognition reaction by monoclonal antibodies against HN [31]. Their sequence analysis has shown that a mutation in amino acid residue 347 from $E$ to $\mathrm{K}$ would change the character of the HN protein and make its escape from epitope recognition by monoclonal antibodies. Cho et al. [32] have reported that the occurrence of E347K mutation in NDV HN protein may reach up to $87.5 \%$. The high prevalence of the mutation could serve as a marker for antigenic variations in NDV.

In general, hygienic treatments and vaccination are two commonly-taken approaches to control NDV spread in commercial farms. The former, i.e. hygienic treatments, includes several techniques such as cleaning, disinfecting, limiting accesses to wild birds, and strict guidance for personal/individual hygienic behavior of farm staffs [33]. The latter, i.e. vaccination, is known to provide an excellent means to decrease chances of clinical disease caused by a virulent NDV [34]. According to Fentie et al. [35], Shabbir et al. [36] and Yune and Abdela [37], a progressive vaccination program should be implemented in combination with biosecurity actions to control the disease. However, vaccination-related policies may vary in different countries [16]. In Indonesia, various vaccination programs have been implemented, which 
consist of a combination of activated and inactivated ND vaccines. A prior study by Wibowo and Amanu [6] has proven that chickens treated with the combination of both types of vaccine are more effectively protected compared to those receiving activated vaccines only. In general, LaSota and B1, which were first developed based on lentogenic NDV strains, are the most commonly used vaccine strains worldwide [38]. In Indonesia, data from the country's Center for Quality Testing and Certification of Veterinary Drugs (BPBPMSOH; Balai Besar Pengujian Mutu dan Sertifikasi Obat Hewan) have shown that vaccines registered in Indonesia include LaSota, B1, RIVS 2, Clone 30, ITA, Mukteswar, Kimber, Ulster, and Genotype 7 (G7) [39]. In general, each of these vaccines is primarily designed to have a full capability in delivering a protection against ND infections if the vaccine is in a viable condition, if it is administered through a proper set of procedures to healthy birds by following applicable guidance, and if there is enough time frame to form an immune response by vaccinated birds to counter an exposure to the virus [40].

\section{Materials and Methods}

\section{Observed samples}

This study investigates field samples taken from laboratory collections, which were previously gathered from various areas and sent to Microbiology Laboratory at the Faculty of Veterinary Medicine, Gadjah Mada University (UGM), Yogyakarta, Indonesia. Among the laboratory collections, a total of nine samples were selected including those gathered from ND outbreak-occurring areas of commercial chicken farms (Table-1).

\section{Isolation of NDV}

Viruses were isolated by applying the OIE 2.3.14 protocol [41]. First, eggs produced by specific-pathogen-free chickens were gathered. When their embryos have been aged 9-11 days, the embryonated eggs were candled to check the viability of embryos. Next, viable eggs were first pre-conditioned in an incubator for 2 days at $37^{\circ} \mathrm{C}$ to put them in good condition before

Table-1: Observed samples in this study.

\begin{tabular}{lllll}
\hline No & Sample code & Origin & Year & Poultry \\
\hline 1 & ND-Lay/KP-145/2013 & Kulonprogo & 2013 & Layer \\
2 & ND-Lay/GK-SR1/2013 & Gunungkidul & 2013 & Layer \\
3 & ND-Lay/GK-SR2/2013 & Gunungkidul & 2013 & Layer \\
4 & ND-Lay/BYL-1/2014 & Boyolali & 2014 & Layer \\
5 & ND-Lay/BYL-3/2014 & Boyolali & 2014 & Layer \\
6 & ND-Lay/ & Magelang & 2014 & Layer \\
& MGL-Pullet-80/2013 & & & \\
7 & ND-Bro/ & Muntilan & 2014 Broiler \\
& MNL-Lingga-2L/2014 & & & \\
8 & ND-Lay/ & Medan & 2014 & Layer \\
& Medan-Pullet-KB/2014 & & & \\
9 & ND-Lay/PLB-147/2014 & Palembang & 2014 & Layer \\
10 & ND-Bro/ & Muntilan & 2014 & Broiler \\
& MNL-Lingga-IP/2014 & & & \\
\hline
\end{tabular}

ND: Newcastle disease being inoculated. In parallel, sample organs (pulmonary and brain) of ND-infected chickens were homogenized using tissue raptor to have a homogeneous form. The supernatant of the homogenized sample organs was taken as inoculating NDV. After that, the pre-conditioned viable eggs were inoculated by injecting the prepared inoculating NDV as much as $0.1 \mathrm{ml}$ to chorioallantoic fluid (CAF) of the eggs using a $1 \mathrm{ml}$ syringe. Next, the inoculated eggs were incubated at $37^{\circ} \mathrm{C}$. Later, a candling process was repeatedly performed over these incubated eggs. The observation on their embryos was conducted once every $24 \mathrm{~h}$. If an observation revealed the embryonic death of an egg, the respective egg was moved and maintained in a refrigerator. After all incubated eggs have shown embryonic deaths, the CAF of each egg was harvested separately.

\section{Virus identification}

Hemagglutination (HA) test

A total of $0.025 \mathrm{ml}$ phosphate buffer saline (PBS) was placed on a plastic-made microtiter plate (U-shaped well). Next, a $0.025 \mathrm{ml}$ of viral suspension was added to the first well. The virus suspension was diluted into the last well by a multichannel micropipette. In each well, a $0.025 \mathrm{ml}$ PBS solution was added. Besides, a total of $0.025 \mathrm{ml}$ of $0.5 \%$ random chicken red blood cells was added to each well. The red blood cells-added plates were placed at room temperature and observed after $30 \mathrm{~min}$. Alternatively, the plates were placed at $20^{\circ} \mathrm{C}$ temperature and observed after $60 \mathrm{~min}$.

\section{Hemagglutination inhibition (HI) test}

For HI test, a $0.025 \mathrm{ml}$ PBS solution was added to a microtiter plate. Wells with a U-shaped base, except for the first well, acted as a 4-HAU control line. Next, a $0.025 \mathrm{ml} \mathrm{ND-specific} \mathrm{antiserum} \mathrm{was} \mathrm{added} \mathrm{to} \mathrm{the}$ first well, which was then diluted two-fold to the next until last wells for the titration using a multichannel micropipette. After that, a $0.0 .25 \mathrm{ml} 4 \mathrm{HA}$ virus/antigen was added into each well. The plates were then placed at room temperature for $30 \mathrm{~min}$. Alternatively, they might be placed at $4^{\circ} \mathrm{C}$ temperature for $60 \mathrm{~min}$. The next step was the addition of $0.025 \mathrm{ml}$ of $0.5 \%$ random chicken red blood cells into each well. The plates were again incubated for $40 \mathrm{~min}$ at room temperature. Alternatively, they might be incubated for $60 \mathrm{~min}$ at $4^{\circ} \mathrm{C}$ temperature. Furthermore, the incubation could be stated as finished after red-blood cell control had been agglutinated.

\section{Reverse transcriptase polymerase chain reaction (RT-PCR)}

RNA extraction was conducted on a $200 \mu 1$ of CAF using Purelink Viral RNA/DNA mini-kit by following suggested protocols from the kit's manufacturer. After that, the RNA extraction results were taken into the RT-PCR process using forward and reverse primers. The process was conducted to amplify the HN sequence of NDV (Table-2). 
In details, the primer was designed to amplify nucleotide residues at positions 659-1353 (amino acid 256-449) of HN protein using AmplifiX software. Amplification areas were selected according to the immunodominant area of $\mathrm{HN}$ protein of NDVs. In particular, amino acid residues at positions 345-353 have been recognized as having a critical role during the antigenic formation of NDV. Primary specificity of the primer was tested according to Blast search from the National Center for Biotechnology Information (NCBI). Next, aforementioned samples and the primer were replicated and transformed into cDNA at $50^{\circ} \mathrm{C}$ for $30 \mathrm{~min}$. After that, the produced cDNA was pre-denaturized at $95^{\circ} \mathrm{C}$ for $2 \mathrm{~min}$. The pre-denaturation step was performed for 1 cycle only. Next, the pre-denaturation results were denaturized at $95^{\circ} \mathrm{C}$ for $15 \mathrm{~s}$. The denaturation results were annealed at $59^{\circ} \mathrm{C}$ for $30 \mathrm{~min}$ and were extended at $68^{\circ} \mathrm{C}$ for 60 $\mathrm{s}$. These three processes (denaturation, annealing and extension) were repeated for 35 cycles. Next, results produced after these 35 cycles were taken to post-extension steps at $72^{\circ} \mathrm{C}$ for $5 \mathrm{~min}$. The post-extension steps were performed for 1 cycle only, and the machine was set to automatically hold at $4^{\circ} \mathrm{C}$ as a safety precaution for preventing undesired damages on the amplified fragment gene. After all of these RT-PCR cycles have been completed, a gel electrophoresis process of the RT-PCR product was performed using a $1.5 \%$ agarose gel with Florosafe Stain DNA stain in a $1 \times$ buffer TBE solution. Next, the RT-PCR amplification product in the gel was visualized using UV transilluminator. Then, the RT-PCR product showing positive results was taken to FIRST BASE Laboratory in Singapore for nucleic acid sequencing using a capillary sequencer.

\section{Data analysis}

From the nucleic acid sequencing, the sequence result/data were received as an electropherogram in the form of ABI file. The data were converted into a txt file containing the nucleic acid sequence. Next, the converted sequence data were assembled. It was followed by searches on NCBI databases to find similar sequence(s). Next, an analysis of the sequencing data was conducted using Molecular Evolution Genetics Analysis (MEGA) software version $7[42,43]$. In details, the sequence searches were followed by a multiple sequence alignment using MEGA7's ClustalW tool. Genetic distance and homology value of amino acids and nucleotide were analyzed by applying "p-distance" and "no. off difference" options in the program. Next, the data were analyzed using ClustalW's "Neighbor-Joining" option to obtain a phylogenetic tree. Then, the replication percentage of branches-forming phylogenetic tree was tested using a bootstrap test on 1000 replications.

\section{Results}

\section{Isolation and identification of NDV}

NDV isolation was performed by injecting the supernatant of homogenized pulmonary and brain organs from suspected chicken samples of NDV into the CAF of specific-pathogen-free, embryonated and viable eggs. The NDV isolates would cause the death of chicken embryos with an inoculation time of approximately $28 \mathrm{~h}$. The CAF from the inoculated chicken eggs was then used for viral identification tests. Apparently, HA tests showed that all samples appeared to have positive results. Next, the samples showing positive were again tested for HI using a specific anti-ND serum. Apparently, the HI tests showed positive results for NDV also. The nine samples under observation, therefore, showed positive in both HA and HI tests. Table-3 provided the results of isolation and virus identification conducted in Microbiology Laboratory at the Faculty of Veterinary Medicine (FKH), Gadjah Mada University (UGM). Furthermore, a molecular test was conducted by implementing RT-PCR method on samples that showed positive results during both HA and HI tests. The electrophoresis results showed the presence of samples' nucleic acid bands (1-9) as being parallel to the positive control (LaSota vaccine, $\mathrm{K}+$ ) at $694 \mathrm{bp}$ (Figure-1).

\section{Sequence analysis on NDV HN fragment gene (amino acids 256-449)}

For nine samples under observation, the amino acid sequence at positions 345-353, which were known as the immunodominant area of $\mathrm{HN}$ gene of NDV, was discovered to be ${ }^{345}$ Proline- ${ }^{346}$ Aspartate${ }^{347}$ Glutamate- ${ }^{348}$ Glutamine-- ${ }^{349}$ Aspartate- ${ }^{350}$ Tyrosine${ }^{351}$ Glutamine- ${ }^{352}$ Isoleucine- ${ }^{353}$ Arginine(PDEQDYQIR). Table-4 provides the composition of amino acid residues at positions $345-353$ of the nine samples under investigation, existing samples of NDV isolates from

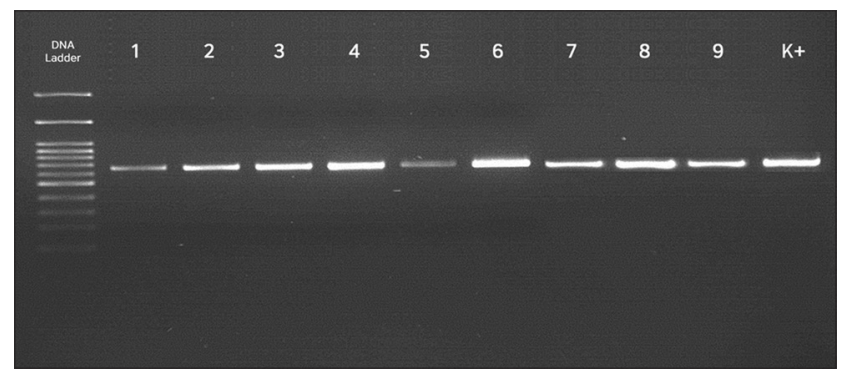

Figure-1: Electrophoresis of $\mathrm{HN}$ gene fragment amplification. Samples 1-9 show positive results, looking at considerably parallel band positions to the control $(\mathrm{K}+)$. In this study, LaSota vaccine strain is posited as the positive control.

Table-2: Designed primers (forward and reverse) at nucleotide positions 659-1353 of HN gene.

\begin{tabular}{lllcr}
\hline Target & Primers & Nucleotide & Length & Positions \\
\hline HN & Forward & 5'GTGAGTGCAACCCCTTTAGGTTGT3' & 694 bp & 659 \\
& Reverse & 5'TAGACCCCAGTGATGCATGAGTTG3' & & 1353 \\
\hline
\end{tabular}

HN: Hemagglutinin-neuraminidase 
Table-3: Vaccination records, clinical signs, the results of HA, HI, and RT-PCR test on observed samples.

\begin{tabular}{|c|c|c|c|c|c|c|}
\hline \multirow[t]{2}{*}{ No } & \multirow[t]{2}{*}{ Samples code } & \multirow[t]{2}{*}{ Vaccination } & \multirow[t]{2}{*}{ Clinical signs } & \multicolumn{3}{|c|}{ Test results } \\
\hline & & & & HA & HI & RT-PCR \\
\hline 1 & ND-Lay/KP-145/2013 & $\begin{array}{l}4 \text { (ND IB live); } \\
18 \text { (ND live); } \\
35 \text { (ND live) }\end{array}$ & $\begin{array}{l}\text { Production drop, torticollis, } \\
\text { mortality } 5 \%\end{array}$ & + & + & + \\
\hline 2 & ND-Lay/GK-SR-1/2013 & $\begin{array}{l}16 \text { weeks, revaccination: } \\
1.5 \text { month }\end{array}$ & Production drop & + & + & + \\
\hline 3 & ND-Lay/GK-SR2/2013 & $\begin{array}{l}16 \text { weeks, revaccination: } \\
1.5 \text { month }\end{array}$ & Production drop & + & + & + \\
\hline 4 & ND-Lay/BYL-1/2014 & $\begin{array}{l}1 \text { (ND IB live); } \\
5 \text { (ND IB killed); } 27 \text { (ND } \\
\text { live); } \\
49 \text { (ND IB live); } \\
70 \text { (ND IB live) }\end{array}$ & Torticollis, high mortality & + & + & + \\
\hline 5 & ND-Lay/BYL-3/2014 & $\begin{array}{l}1 \text { (ND IB live); } \\
5 \text { (ND IB killed); } \\
27 \text { (ND live); } \\
49 \text { (ND IB live); } \\
70 \text { (ND IB live) }\end{array}$ & Torticollis, high mortality & + & + & + \\
\hline 6 & ND-Lay/MGL-Pullet-KB/2014 & $\begin{array}{l}4 \text { (ND IB); } \\
21 \text { (ND live); } \\
56 \text { (ND IB) }\end{array}$ & $\begin{array}{l}\text { Production drop, } \\
\text { High mortality }\end{array}$ & + & + & + \\
\hline 7 & ND-Bro/MNL-Lingga-2L/2014 & $\begin{array}{l}4 \text { (ND IB live); } \\
21 \text { (ND) }\end{array}$ & High mortality & + & + & + \\
\hline 8 & ND-Lay/PLB-147/2014 & $\begin{array}{l}5 \text { (ND live); } \\
16 \text { (ND live); } \\
28 \text { (ND live); } \\
35 \text { (ND killed) }\end{array}$ & High mortality & + & + & + \\
\hline 9 & ND-Lay/Medan-Pullet-KB/2014 & $\begin{array}{l}1 \text { (ND IB); } \\
4 \text { (ND IB killed); } \\
20 \text { (ND live) }\end{array}$ & High mortality & + & + & + \\
\hline
\end{tabular}

HA: Hemagglutination, HI: Hemagglutination inhibition, RT-PCR: Reverse transcriptase-polymerase chain reaction, ND: Newcastle disease

Table-4: Immunodominant area from observed samples, previously-recorded isolates from Indonesia in GenBank, and the LaSota strain.

\begin{tabular}{llll}
\hline Virus & Genbank accession number & Origin & Residue (345-353) \\
\hline Samples & & & \\
\hline ND-Lay/KP-145/2013 & - & Kulonprogo & PDEQDYQIR \\
ND-Lay/GK-SR-1/2013 & - & Gunung Kidul & PDEQDYQIR \\
ND-Lay/GK-SR2/2013 & - & Gunung Kidul & PDEQDYQIR \\
ND-Lay/BYL-1/2014 & - & Boyolali & PDEQDYQIR \\
ND-Lay/BYL-3/2014 & - & Boyolali & PDEQDYQIR \\
ND-Lay/MGL-Pullet-KB/2014 & - & Magelang & PDEQDYQR \\
ND-Bro/MNL-Lingga-2L/2014 & - & Muntilan & PDEQDYQR \\
ND-Lay/PLB-147/2014 & - & Palembang & PDEQDYQIR \\
ND-Lay/Medan-Pullet-KB/2014 & - & Medan & PDEQDYQIR \\
\hline Previous Isolates from & & & \\
Indonesia & & & PDEQDYQIR \\
\hline NDV/Cockatoo/Indonesia/14698/90 & AY288998 & PDEQDYQIR \\
NDV/Chicken/Bali/020/10 & HQ697261 & Bdonesia & PDEQDYQIR \\
NDV/Chicken/Banjarmasin/010/10 & HQ697254 & Banjarmasin & PDEQDYIR \\
NDV/Chicken/Gianyar/013/10 & HQ697257 & Bali & PDEQ \\
NDV/Chicken/Kudus/017/10 & HQ697259 & PDEQ \\
NDV/Chicken/Kudus/018/10 & HQ697260 & Pudus \\
NDV/Chicken/Makassar/003/09 & HQ697256 & Makassar & PDEQDYQIR \\
NDV/Chicken/Sragen/014/10 & HQ697258 & Sragen & PDEQDYQR \\
NDV/Chicken/Sukorejo/019/10 & HQ697255 & Sukorejo & \\
NDV/Chicken/Jakarta/1997 & JX393313 & Jakarta & \\
\hline Vaccine & & & PDEQDYQIR \\
\hline LaSota & FJ004152 & &
\end{tabular}

ND: Newcastle disease, NDV: Newcastle disease virus 
Indonesia previously recorded in the GenBank database, and existing vaccine strains used in Indonesia. It appeared that all of these current, existing and vaccine samples have the same amino acid sequence of PDEQDYQIR. The complete comparison of amino acid changes in individual virus isolates is provided in Table-5.

\section{Phylogenetic analysis of NDV}

Phylogenetic analysis was performed on the fragment of NDV HN gene sequence. Looking at phylogenetic tree produced by an analysis on amino acids at positions 256-449, all isolates observed in this study, which were gathered from Kulon Progo, Gunung Kidul (2), Boyolali (2), Magelang, Muntilan (2), Palembang and Medan, were discovered to have a close relation with existing NDVs that had infected poultry farms in some regions in Indonesia, i.e. Banjarmasin (Accession: HQ697254), Gianyar (Accession: HQ697257), Kudus (Accession: HQ697259, HQ697260) and Sragen (Accession: HQ697258).

\section{Genetic distance and homology between HN gene fragments}

Amino acids analyses on the nine virus isolates under observation in this study showed that their genetic distance was $0-1.5 \%$ with homology values at 98.4-100\%. On the other hand, the genetic distance of amino acids between the nine virus isolates compared to previously recorded isolates from Indonesia (GenBank database) was $0-10.8 \%$ with homology values at $89.1-100 \%$. Then, comparing NDV isolates under investigation with existing LaSota vaccine strain applied in Indonesia showed their amino acid genetic distance in between $8.2 \%$ and $8.8 \%$ with homology values at $91.2-91.7 \%$. On the other hand, a nucleotide analysis on the nine virus isolates provided $0-1.2 \%$ genetic distance with homology values at $98.8-100 \%$. Besides, the nucleotide distance between the observed virus isolates compared to previously recorded isolates from Indonesia (GenBank) was $0-7.7 \%$ with homology values at $92.3-99.8 \%$. Then, comparing isolated NDV under investigation with existing LaSota vaccine strain discovered their nucleotide genetic distance to be in between 16.7 and $17 \%$ with $83.03-83.33 \%$ homology values.

\section{Discussion}

Virus isolation remains the definitive diagnostic evaluation for NDV [41]. In this study, the nine virus isolates under observation were classified into the velogenic NDV group [1] because the embryonic deaths of inoculated egg samples were discovered to occur in less than $60 \mathrm{~h}$. On the other hand, the HA and HI tests were performed as conventional evaluations for NDV identification. In fact, both HA and HI tests showed positive results for all samples. In particular, the positive results $(+)$ in HA test were observed by discovering the agglutination of poultry red-blood cells at the bottom of observed wells plate. The agglutination could occur because the red blood cells were bound into virus particles. The process was assisted by hemagglutinin protein on the surface of the NDV. The protein has been recognized as having an ability to agglutinate red-blood cells [44]. In parallel, positive results discovered by HI tests were discovered by observing the formation of red blood cell precipitates at the bottom of the plate. These precipitates were formed due to the binding of viral suspension into specific serum of NDV. The binding process has been known to cause the NDV to not being able to agglutinate the red blood cells.

Furthermore, the RT-PCR procedures were conducted to identify virus from observed samples based on a molecular technique. Amplification was performed on $\mathrm{HN}$ gene fragments using primers designed in AmplifiX software. The presence of DNA bands, which appeared to be nearly parallel to the positive control (LaSota vaccine) at around position $694 \mathrm{bp}$, showed that the nine test samples were molecularly proven to be NDV. Besides, the pattern of amino acid

Table-5: Differences between amino acid from isolate samples compared to vaccine strain LaSota.

\begin{tabular}{|c|c|c|c|c|c|c|c|c|c|c|c|c|c|c|c|c|c|}
\hline \multirow[t]{2}{*}{ No } & \multirow[t]{2}{*}{ Isolates } & \multicolumn{16}{|c|}{ Amino acid positions } \\
\hline & & 265 & 267 & 268 & 271 & 284 & 290 & 292 & 295 & 310 & 312 & 317 & 331 & 371 & 389 & 397 & 433 \\
\hline \multicolumn{18}{|c|}{ Vaccine strain } \\
\hline 1 & FJ004152_ND_LaSota & $\mathrm{N}$ & A & $\mathrm{V}$ & $\mathrm{R}$ & $\mathrm{H}$ & $\mathrm{V}$ & $\mathrm{T}$ & G & $\mathrm{I}$ & $\mathrm{S}$ & $\mathrm{S}$ & $\mathrm{V}$ & I & V & V & V \\
\hline \multicolumn{18}{|c|}{ Isolate samples } \\
\hline 2 & $\begin{array}{l}\text { ND-Bro/ } \\
\text { MNL-Lingga-2L/2014 }\end{array}$ & $\mathrm{K}$ & I & A & $\mathrm{S}$ & $\mathrm{R}$ & $\mathrm{T}$ & A & $\mathrm{K}$ & V & $\mathrm{E}$ & $\mathrm{P}$ & A & V & M & I & I \\
\hline 3 & ND-Lay/BYL-1/2014 & $\mathrm{K}$ & $\mathrm{V}$ & $A$ & $\mathrm{~S}$ & $\mathrm{H}$ & $\mathrm{T}$ & $A$ & $E$ & V & $\mathrm{E}$ & $\mathrm{P}$ & $A$ & V & M & $\mathrm{I}$ & $\mathrm{I}$ \\
\hline 4 & ND-Lay/BYL-3/2014 & $\mathrm{K}$ & I & A & $\mathrm{S}$ & $\mathrm{R}$ & $\mathrm{T}$ & $A$ & $\mathrm{~K}$ & V & $E$ & $\mathrm{P}$ & $A$ & V & M & $\mathrm{I}$ & $\mathrm{I}$ \\
\hline 5 & ND-Lay/GK-SR1/2013 & $\mathrm{K}$ & V & A & $\mathrm{S}$ & $\mathrm{H}$ & $\mathrm{T}$ & $A$ & $E$ & V & $\mathrm{E}$ & $\mathrm{P}$ & $A$ & V & M & $\mathrm{I}$ & $\mathrm{I}$ \\
\hline 6 & ND-Lay/GK-SR2/2013 & $\mathrm{K}$ & V & A & $\mathrm{S}$ & $\mathrm{H}$ & $\mathrm{T}$ & $A$ & $E$ & V & $E$ & $\mathrm{P}$ & $A$ & V & M & $\mathrm{I}$ & $\mathrm{I}$ \\
\hline 7 & ND-Lay/KP-145/2013 & $\mathrm{K}$ & V & A & $\mathrm{S}$ & $\mathrm{H}$ & $\mathrm{T}$ & A & $E$ & V & $\mathrm{E}$ & $\mathrm{P}$ & A & V & M & $\mathrm{I}$ & $\mathrm{I}$ \\
\hline 8 & $\begin{array}{l}\text { ND-Lay/ } \\
\text { Medan-Pullet-KB/2014 }\end{array}$ & $\mathrm{K}$ & V & A & $\mathrm{S}$ & $\mathrm{H}$ & $\mathrm{T}$ & A & $E$ & V & $\mathrm{E}$ & $\mathrm{P}$ & A & $\mathrm{V}$ & M & $\mathrm{I}$ & $\mathrm{I}$ \\
\hline 9 & $\begin{array}{l}\text { ND-Lay/ } \\
\text { MGL-Pullet-80/2013 }\end{array}$ & $\mathrm{K}$ & V & A & $\mathrm{S}$ & $\mathrm{H}$ & $\mathrm{T}$ & A & $E$ & V & $\mathrm{E}$ & $\mathrm{P}$ & A & V & M & $\mathrm{I}$ & $\mathrm{I}$ \\
\hline 10 & ND-Lay/PLB-147/2014 & $\mathrm{K}$ & $\mathrm{I}$ & $A$ & $\mathrm{~S}$ & $\mathrm{R}$ & $\mathrm{T}$ & $A$ & $\mathrm{~K}$ & V & $E$ & $P$ & $A$ & V & M & $\mathrm{I}$ & $\mathrm{I}$ \\
\hline
\end{tabular}

ND: Newcastle disease 
residues at positions $345-353$ of either the nine samples being tested, existing NDV isolates from Indonesia in the GenBank database, or the LaSota vaccine strains have the same amino acid sequence of PDEQDYQIR. The amplification of the amplified area was implemented according to prior researches conducted by Iorio et al. [29], $\mathrm{Hu}$ et al. [31], and $\mathrm{Gu}$ et al. [45]. These studies have suggested that the amino acid residue at positions $345-353$ in the NDV HN protein was a critical immunodominant area during an antibody recognition process. There was, in fact, no significant difference regarding amino acid sequence between viral isolates and existing vaccine strains applied in Indonesia. Miller and Koch [46] have reported that a matched vaccine strain to an outbreak has the highest potential to provide superior protection against virus transmissions. However, multiple factors might decrease the effectiveness of vaccination. According to Nakamura et al. [47], a vaccine failure might occur due to numerous factors occurred within a host (immunosuppressive condition), in a vaccine itself (improper vaccination) and the occurrence of pathogenic virus. To complement a vaccination program, controlling NDV should be supported by a strict biosecurity system to holistically prevent the virus from ever in contact with a poultry farm [46]. Furthermore, previous studies by Iorio et al. [29], Hu et al. [31] and Gu et al. [45] on the immunodominant nature of $\mathrm{HN}$ protein have shown that existing vaccines used in Indonesia should be protective against any NDV outbreak. The absence of mutations at the immunodominant areas of NDV has caused antibodies of individual hosts produced by the vaccine to recognize the immunodominant areas [31]. Theoretically, the recognition of a host's antibodies to the immunodominant region would lead to HA processes. The process of viral binding with the host's cell receptors could hence be avoided. However, the protective characteristics of LaSota vaccine, in addition to evaluation on the amino acid sequence within an antibody recognition site, should also be evaluated from the genetic and homologous distances between a circulating virus and the vaccine.

Moreover, both $\mathrm{HN}$ and $\mathrm{F}$ proteins have been noted as being the main targets for the formation of different ND vaccines [46]. Besides, these two proteins have been posited as the primary target of a host's immune response to ND. In particular, HN proteins would induce an immune response as the host's body activated its defense against a viral infection. Therefore, the protein could easily produce an antigenic variation in NDV when confronted with the host's immune system $[38,48]$. Cho et al. [32] have reported that the prevalence of amino acid residues of E347K in NDV occurred to be at $87.5 \%$. Most of which were found in isolates circulating in Korea and China mainland and included in the genotype II and VII of NDVs. According to Zhu et al. [49], LaSota has been discovered to not being able to provide complete protection against an E347K infection. Hence, LaSota-vaccinated birds were still able to get infected and to shed the virulent challenge (infecting) virus. In this study, however, no isolate experienced any mutation on E347K amino acid residues.

Looking at the phylogenetic tree produced by an analysis on amino acids (Figure-2), all nine isolates observed in this study, which were gathered from Kulon Progo, Gunung Kidul (2), Boyolali (2), Magelang, Muntilan (2), Palembang and Medan

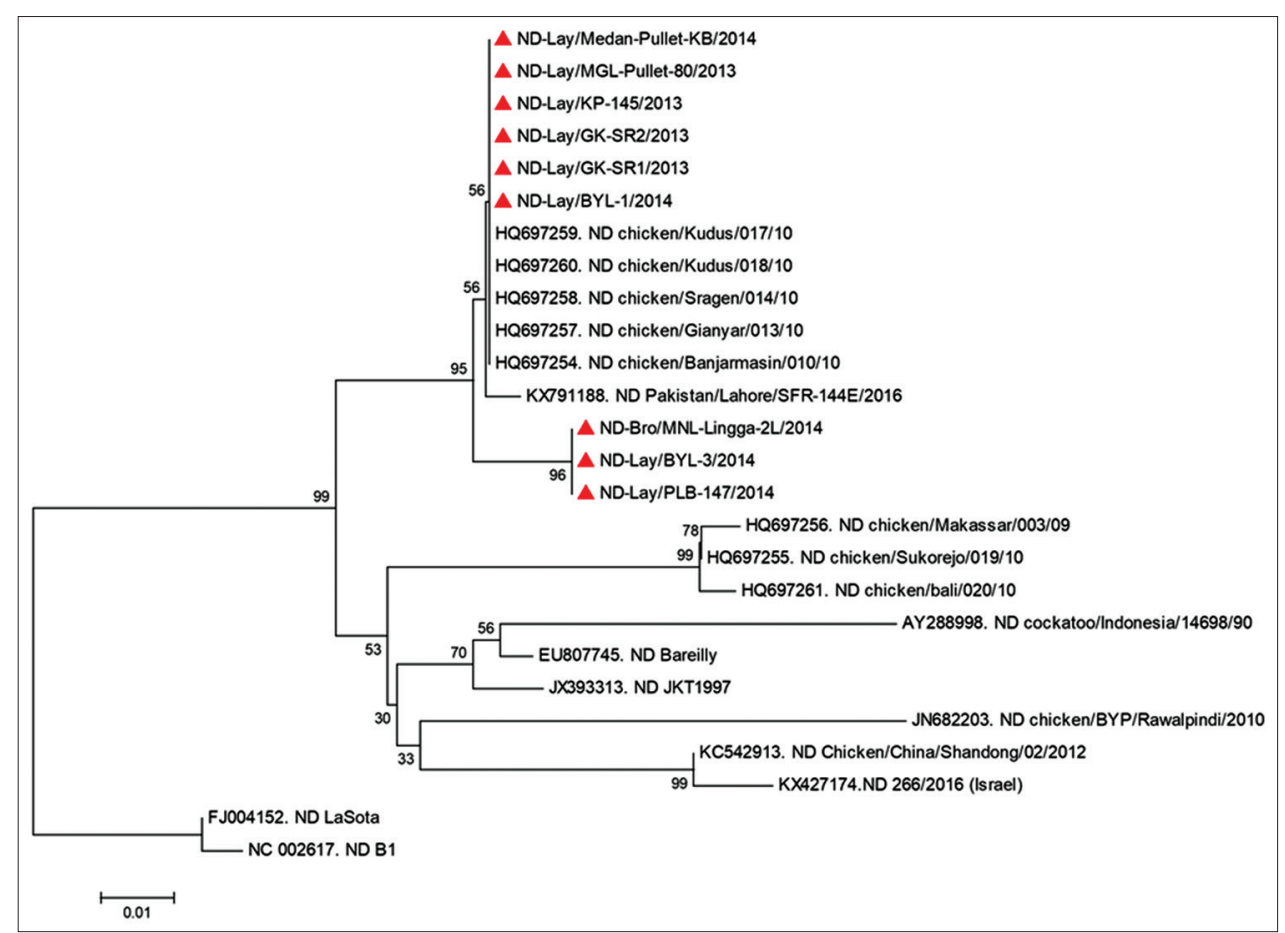

Figure-2: Phylogenetic tree of the HN fragment of the Newcastle disease virus based on amino acid structure at positions 256-449. 
regions, have a close relation to previously-reported NDV isolates from Indonesia, which were recorded in the GenBank database. The existing isolates included that of Banjarmasin (Accession: HQ697254), Gianyar (Accession: HQ697257), Kudus (Accession: HQ697259, HQ697260) and Sragen (Accession: HQ697258). The last reported infection of these existing virus isolates occurred in 2010 [13]. Besides, an analysis on F gene conducted by Xiao et al. [13] has indicated that NDV isolated from Banjarmasin, Gianyar, Kudus and Sragen were classified into genotype VII. In addition, the nine observed virus samples also appeared to be closely related to isolates originated from Pakistan (Accession: KX427174), which was reported by Reihmani et al. [50] as having caused outbreaks in the country. Based on an analysis of its F genes, the Pakistan isolate was classified into genotype VII $[50,51]$. On the other hand, LaSota was classified as a strain of genotype II. Liu et al. [52] and Jeon et al. [53] have reported the LaSota (genotype II) vaccines (both live and killed vaccines) to deliver effective protection against viruses originated from different genotypes. However, Dimitry et al. [12] and Liu et al. [52] have reported vaccines that were phylogenetically close to outbreak-causing NDVs to provide better protection against ND. In particular, protection as such was observed to occur in terms of reducing the spread of NDV by infected individuals. Although conventional vaccines (LaSota) have been noted to work well in protecting poultry, it could not perfectly prevent infections and virus shedding from occurring [54,55].

Then, genetic distances discovered in this study would arguably affect the efficacy of any ND vaccination. In other words, it might alter a general consensus in which vaccines that were genetically matched with outbreaks virus would result in better protection against ND disease. In details, the lower the genetic distance between a virus on the field and a vaccine strain, protection produced against ND should be better. In this study, an investigation on the homology values between observed virus and LaSota vaccine strain revealed a considerably distant result compared to a perfect condition in which the vaccine had been tested as a perfect and consistent solution $[12,56]$. In short, a distance to the perfect condition has indicated a potential to allow an ND outbreak to occur even in periodically vaccinated commercial chicken farms. The argument has in fact supported by Yang et al. [57], Ewies et al. [58], Garcia et al. [59] and Mohamed et al. [60] who have noted that any genetic variation between a vaccine strain and a circulating NDV might cause the inability of currently used vaccine to deliver a protection against ND.

\section{Conclusion}

Looking at the results of performed HN protein fragment analysis in this study, NDVs isolated from regularly-vaccinated commercial chicken farms appear to have an amino acid structure of PDEQDYQIR at residue positions 345-353. Besides, an analysis on the phylogenetic tree of the nine observed isolates has revealed a close relation to several previously recorded isolates from Indonesia (Banjarmasin, Gianyar, Kudus and Sragen) and Pakistan. Looking at various well-established vaccination programs implemented in chicken farms in Indonesia and at the amino acids structure of circulating NDVs, this study indicates that existing LaSota vaccines should be able to deliver effective protection against ND outbreaks in well-vaccinated commercial chicken farms. However, the discovered genetic distance between currently circulating virus and the LaSota vaccine strain might have acted as the probable root cause of ND outbreaks occurring in commercial chicken farms despite the regular implementation of various vaccination programs. The argument being proposed has in fact been supported by prior researchers. Those studies have underlined as small as possible differences between current virus strain condition by which a virus is circulating with the perfect virus strain condition to which a vaccine was first developed to deliver perfect protection as a critical cause of vaccination failures.

Then, prior study by Sarcheshmei et al. [61] has reported that any vaccination failure against a circulating NDV might also occur due to other factors such as the presence of immunosuppressive etiologic agents causing a lack of or inadequate responses to the vaccine; vaccination failures including an improper handling of vaccine administration; poor management practices in poultry flocks, etc. Therefore, further researches may put these factors as the focuses of studies to complement the current study that has suggested the lack of potency of a vaccine product as the probable root cause of vaccination failures.

\section{Authors' Contributions}

LST worked out a major part of the technical details, performed laboratory and computational preparations, tests as well as analyses, and took the lead in writing the manuscript in consultation with MHW and RW. MHW devised the project, conceived the research design, handled collaborative works with external partners, verified computational framework, and verified the results of laboratory and computational tests as well as data analyses. RW designed primers and computational framework, verified laboratory and data analysis methods and analyzed test results conducted by external partners. All authors read and approved the final manuscript.

\section{Acknowledgments}

This research was financially supported by Biosafety Engagement Program, Agricultural Research Service of the United States Development Agency (ARS-USDA), CDRF Project 60072/2014. 


\section{Competing Interests} interests.

The authors declare that they have no competing

\section{References}

1. Alexander, D.J., Aldous, E.W. and Chad, M.F. (2012) The long view: A selected review of 40 years of Newcastle disease research. Avian Pathol., 41: 329-335.

2. Sa'idu, L. and Abdu, P.A. (2008) Outbreak of viscerotropic velogenic form of Newcastle disease in vaccinated six weeks old pullets. Sokoto J. Vet. Sci., 7: 37-40.

3. OIE-World Organisation for Animal Health. (1996) Newcastle disease. In: Manual of Standards for Diagnostic Test and Vaccines. $3^{\text {rd }}$ ed. OIE, Paris. p161-169.

4. Kencana, G.A.Y. (2012) Penyakit Virus Unggas. Udayana University Press, Denpasar. p30-40.

5. Darminto, S.M. (2005) Kajian Newcastle Disease pada itik dan upaya pengendaliannya. Wartazoa, 15: 84-94.

6. Wibowo, M.H. and Amanu, S. (2010) Perbandingan beberapa program vaksinasi penyakit Newcastle pada ayam buras. J. Sains Vet., 28: 27-35.

7. Dharmayanti, I., Hartawan, R., Hewajuli, D.A. and Indriani, R. (2014) Phylogenetic analysis of genotype VII of Newcastle Disease virus in Indonesia. Afr. J. Microbiol. Res., 8: 1368-1374.

8. Kraneveld, F.C. (1926) A poultry disease in the Dutch East Indies, Nederlands-Indische. Bl. Voor Diergeneeskd., 38: 448-451.

9. Doyle, T.M. (1927) A hit her to unrecorded disease of fowl due to a filter passing virus. J. Comp. Pathol. Ther, 40: 144-169.

10. Ashraf, A. and Shah, M. (2014) Newcastle disease: Present status and future challenges for developing countries. Afr. $J$. Microbiol. Res., 8: 411-416.

11. Naveen, K.A., Singh, S.D., Kataria, J.M., Barathidasan, R. and Dhama, K. (2013) Detection and differentiation of pigeon paramyxovirus serotype-1(PPMV-1) isolates by RT-PCR and restriction enzyme analysis. Trop. Anim. Health Pro., 45: 1231-1236.

12. Dimitrv, K.M., Afonso, C.L., Yu, Q. and Miller, P.J. (2017) Newcastle disease vaccines-A solved problem or a continuous challenge? Vet. Microbiol., 206: 126-136.

13. Xiao, S., Paldurai, A., Nayak, B., Samuel, A., Bharoto, A.E., Prajitno, T.Y., Collins, P.L. and Samal, S.K. (2012) Complete genome sequences of Newcastle disease virus strains circulating in chiken populations of Indonesia. J. Virol., 86: 5969-5970.

14. Darminto and Ronohardjo, P. (1996) Newcastle Disease pada Unggas di Indonesia: Situasi Terakhir dan Relevansinya terhadap Pengendalian Penyakit. Balai Penelitian Veteriner. p65-84.

15. Cattoli, G., Susta, L., Terregino, C. and Brown, C. (2011) Newcastle disease: A Shahid and Liaquat 366 review of field recognition and current methods of laboratory detection. J. Vet. Diagn. Invest., 23: 637-656.

16. Miller, P.J. and Koch, G. (2013) Newcastle disease. In: Swayne, D.E., Glisson, J.R., McDougald, L.R., Nolan, L.K., Suarez, D.L.O. and Nair, V.N., editors. Diseases of Poultry. $13^{\text {th }}$ ed. John Wiley \& Sons, Athens. p89-107.

17. Lamb, R.A. and Parks, G.D. (2007) Paramyxoviridae: The viruses and their replication. In: Fields, B.N., Knipe, D.N. and Howley, P.M., editors. Fields Virology. $5^{\text {th }}$ ed. Lippincott, Williams, and Wilkins, Philadelphia. p1449-1496.

18. Cao, Y., Gu, M., Zhang, X., Liu, W. and Liu, X. (2013) Complete genome sequences of two Newcastle disease virus strains of genotype VIII. J. Genome Announcement, 1: e00180-12.

19. Zhang, Y.Y., Shao, M.Y., Yu, X.H., Zhao, J. and Zhang, G.Z. (2014) Molecular characterization of chicken-derived genotype VIId Newcastle Disease virus isolates in China during
2005-2012 reveals a new length in hemagglutinin-neuraminidase. Infect. Genet. Evol., 21: 359-366.

20. Panda, A., Huang, Z., Elankumaran, S., Rockemann, D.D. and Samal, S.K. (2004) Role of fusion protein cleavage site in the virulence of Newcastle disease virus. Microb. Pathog., 36: 1-10.

21. Connaris, H., Takimoto, T., Russell, R., Crennell, S., Moustafa, I., Portner, A. and Taylor, G. (2002) Probing the sialic acid binding site of the hemagglutinin-neuraminidase of Newcastle disease virus: Identification of key amino acids involved in cell binding, catalysis and fusion. J. Virol., 76: 1816-1824.

22. Connolly, S.A., Leser, G.P., Jardetzky, T.S. and Lamb, R.A. (2009) Bimolecular complementation of paramyxovirus fusion and hemagglutinin-neuraminidase proteins enhances fusion: Implications for the mechanism of fusion triggering. J. Virol., 83: 10857-10868.

23. Kim, S.H., Subbiah, M., Samuel, A.S., Collins, P.L. and Samal, S.K. (2011) Roles of the fusion and hemagglutinin-neuraminidase proteins in replication, tropism, and pathogenicity of avian paramyxoviruses. J. Virol., 85: 8582-8596

24. Huang, Z., Panda, A., Elankumaran, S., Govindarajan, D., Rockemann, D.D. and Samal, S.K. (2004) The hemagglutinin-neuraminidase protein of Newcastle disease virus determines tropism and virulence. J. Virol., 78: 4176-4184.

25. Porotto, M., Salah, Z., DeVito, I., Talekar, A., Palmer, S.G., Xu, R., Wilson, I.A. and Moscona, A. (2012) The second receptor binding site of the globular head of the Newcastle disease virus hemagglutinin-neuraminidase activates the stalk of multiple paramyxovirus receptor binding proteins to trigger fusion. J. Virol., 86: 5730-5741.

26. Heiden, S., Grund, C., Röder A., Granzow, H., Kühnel, D., Mettenleiter, T.C. and Romer-Obedorfer, A. (2014) Different regions of the Newcastle Disease virus fusion protein modulate pathogenicity. PLoS One, 9: e113344.

27. Murulitharan, K., Yusoff, K., Omar, A.R. and Molouki, A. (2013) Characterization of Malaysian velogenic NDV strain AF2240-I genomic sequence: A comparative study. Virus Genes, 46: 431-440.

28. Jin, J., Zhao, J., Ren, Y., Zhong, Q. and Zhang, G. (2016) Contribution of HN protein length diversity to Newcastle Disease virus virulence, replication and biological activities. Sci. Rep., 6: 36890.

29. Iorio, R.M., Syddall, R.J., Sheehan, J.P., Bratt, M.A., Glickman, R.L. and Riel, A.M. (1991) Neutralization map of the hemagglutinin-neuraminidase glycoprotein of Newcastle Disease virus: Domains recognized by monoclonal antibodies that prevent receptor recognition. J. Virol., 65: 4999-5006.

30. Mahon, P.J., Mirza, A.M. and Iorio, R.M. (2011) Role of the two sialic acid binding sites on the Newcastle Disease virus HN Protein in triggering the interaction with the $\mathrm{F}$ protein required for the promotion of fusion. J. Virol., 85: 12079-12082.

31. Hu, S., Ma, H., Wu, Y., Liu, W., Wang, X., Liu, Y. and Liu, X. (2010) A vaccine candidate of attenuated genotype VII Newcastle Disease virus generated by reverse genetics. Vaccine, 27: 904-910.

32. Cho, S.H., Kwon, H.J., Kim, T.E., Kim, J.H., Yoo, H.S., Park, M.H., Park, Y.H. and Kim, S.J., (2008) Characterization of a recombinant Newcastle Disease vaccine strain. Clin. Vaccine Immunol., 15: 1572-1579.

33. Kang, Y., Li, Y., Yuan, R., Sun, M., Wang, Z., Feng, M., Jiao, P. and Ren, T. (2014) Phylogenetic variation of two Newcastle Disease viruses isolated from domestic ducks in Southern China. Virol. J., 11: 147.

34. Kapczynski, D.R. and King, D.J. (2005) Protection of chickens against overt clinical disease and determination of viral shedding following vaccination with commercially available Newcastle Disease virus vaccines upon challenge with highly virulent virus from the California 2002 exotic 
Newcastle disease outbreak. Vaccine, 23: 3424-3433.

35. Fentie, T., Dadi, K., Kassa, T., Sahle, M. and Cattoli, G. (2014) Effect of vaccination on transmission characteristics of highly virulent Newcastle disease virus experimentally infected chickens. Avian Pathol., 43: 420-426.

36. Shabbir, M.Z.,Zohari, S., Yaqub, T., Nazir, J., Shabbir, M.A.B., Mukhtar, N., Shafee, M., Sajid, M., Anees, M., Abbas, M., Khan, M.T. Ali, A.A., Ghafoor, A., Ahad, A., Chana, A.A., Anjum, A.A., Hussain, N., Ahmad, A., Goraya, M.U., Iqbal, Z., Khan, S.A., Aslam, H., Zehra, K., Sohail, M.U., Yaqub, W., Ahmad, N., Berg, M. and Munir, M. (2013) Genetic diversity of Newcastle Disease virus in Pakistan: A countrywide perspective. Virol. J., 10: 170.

37. Yune, N. and Abdela, N. (2017) Update on epidemiology, diagnosis and control technique of Newcastle Disease. $J$. Vet. Sci. Tech., 8: 429.

38. Roohani, K., Tan, S.W., Yeap, S.K., Ideris, A., Bejo, M.H. and Omar, A.R. (2015) Characterization of genotype VII Newcastle Disease virus (NDV) isolated from NDV vaccinated chickens and the efficacy of LaSota and recombination genotype VII vaccines against challenge with velogenic NDV. J. Vet. Sci., 16: 447-457.

39. Emilia, A., Setyaningsih, S. and Soejoedono, R.D. (2015) Isolasi dan karakteristik biologik virus Newcastle Disease. J. Kedokteran Hewan, 9: 47-51.

40. Cornax, I., Miller, P.J. and Afonso, C.L. (2012) Characterization of live LaSota vaccine strain-induced protection in chickens upon early challenge with a virulent Newcastle disease virus of heterologous genotype. Avian Dis., 56: 464-470.

41. OIE-World Organisation for Animal Health, (2012) Newcastle disease. In: Manual of Diagnostic Tests and Vaccines for Terrestrial Animals: Mammals, Birds and Bees. Biological Standards Commission-World Organisation for Animal Health, Paris, France. p555-574.

42. Kumar, S., Tamura, K. and Nei, M. (1994) MEGA: Molecular evolutionary genetics analysis software for microcomputers. Comput. Appl. Biosci., 10: 189-191.

43. Kumar, S., Glen, S. and Koichiro, T. (2016) MEGA7: Molecular evolutionary genetics analysis version 7.0 for bigger datasets. Mol. Biol. Evol., 33: 1870-1874.

44. Cosset, F.L., Bouquet, J.F., Drynda, A., Chebloune, Y., ReySenelonge, A., Kohen, G., Nigon, V.M., Desmettre, P. and Verdier, G. (1991) Newcastle Disease virus (NDV) vaccine based on immunization with avian cells expressing the NDV-hemagglutinin-neuraminidase glycoprotein. Virology, 185: 862-866.

45. Gu, M., Liu, W., Xu, L., Cao, Y., Yao, C., Hu, S. and Liu, X. (2011) Positive selection in the hemagglutinin-neuraminidase gene of Newcastle Disease virus and its effect on vaccine efficacy. Virol. J., 8: 150.

46. Miller, P.J. and Koch, G. (2013) Newcastle disease. In: Swayne, D.E., Glisson, J.R., McDougald, L.R., Nolan, L.K., Suarez, D.L. and Nair, V., editors. Diseases of Poultry. $13^{\text {th }}$ ed. Wiley-Blackwell, Hoboken, NJ. p89-138.

47. Nakamura, K., Ito, M., Nakamura, T., Yamamoto, Y., Yamada, M., Mase, M. and Imai, K. (2014) Pathogenesis of Newcastle disease in vaccinated chickens: Pathogenicity of isolated virus and vaccine effect on challenge of its virus. $J$. Vet. Med. Sci., 76: 31-36.

48. Liu, X.F., Wan, H.Q., Ni, X.X., Wu, Y.T. and Liu, W.B. (2003) Pathotypical and genotypical characterization of strains of Newcastle Disease virus isolated from outbreaks in chicken and goose flocks in some regions of China during 1985-2001. Arch. Virol., 148: 1387-1403.

49. Zhu, J., Hu, S., Xu, H., Liu, J., Zhao, Z., Wang, X. and Liu, X. (2016) Characterization of virulent Newcastle Disease viruses from vaccinated chicken flocks in Eastern China. BMC Vet. Res., 12: 113.

50. Rehmani, S. F., Wajid, A., Basharat, A., Bibi, T. and Arif, S. (2016) Newcastle disease virus isolate backyard/Pakistan/ Lahore/SFR-144E/2016, complete genome. Gen Bank, 2016: Accession KX791188.1.

51. Habib, H., Reihmani, S.F., Mukhtar, N., Bibi, T. and Wajid, A. (2015) Biological and molecular characterization of Newcastle Disease virus through haemaglutinin-neuraminidase gene isolated from Lahore district. J. Inf. Mol. Biol., 3: 28-33.

52. Liu, J., Zhu, J., Xu, H., Li, J., Hu, Z., Hu, S. and Liu, X. (2017) Effects of the HN antigenic difference between the vaccine strain and the challenge strain of Newcastle Disease virus on virus shedding and transmission. Viruses, 9: 225.

53. Jeon, W.J., Lee, E.K., Lee, Y.J., Jeong, O.M., Kim, Y.J., Kwon, J.H. and Choi, K.S. (2008) Protective efficacy of commercial inactivated Newcastle disease virus vaccines in chickens against a recent Korean epizootic strain. J. Vet. Sci., 9: 295-300.

54. Samuel, A., Nayak, B., Paldurai, A., Xiao, S., Aplogan, G.L., Awoume, K.A., Webby, R.J., Ducates, M.F., Collins, P.L. and Samal, S.K. (2013) Phylogenetic and pathotypic characterization of Newcastle Disease viruses circulating in West Africa and efficacy of a current vaccine. J. Clin. Microbiol., 51: 771-781.

55. Byarugaba, D.K., Mugimba, K.K., Omony, J.B., Oktiwi, M., Wanyana, A., Otim, M.O., Kirunda, H., Nakavuma, J.L., Teillaud, A., Paul, M.C. and Ducatez, M.F. (2014) High pathogenicity and low genetic evolution of avian paramyxovirus type I (Newcastle Disease virus) isolated from live bird markets in Uganda. Virol. J., 11: 173.

56. Wang, J., Yan, L., Zhang, Y., Zhao, Y., Castellan, D., Liu, H. and Wang, Z. (2016) Genomic characterization of a Newcastle Disease virus isolated from ducks in live bird markets in China. PLoS One, 11: e0158771.

57. Yang, H.M., Zhao, J., Xue, J., Yang, Y. Zhang, G. (2017) Antigenic variation of LaSota and genotype VII Newcastle Disease virus (NDV) and their efficacy against challenge with velogenic NDV. Vaccine, 35: 27-32.

58. Ewies, S.S., Ali, A., Tamam, S.M. and Madbouly, H.M. (2017) Molecular characterization of Newcastle disease virus (genotype VII) from broiler chickens in Egypt. Beni Suef Univ. J. Basic Appl. Sci., 6: 232-237.

59. Garcia, S.C., Diel, D.G., Susta, L., Decanini, E.L., Yu, Q., Brown, C.C., Miller, P.J. and Afonso, C.L. (2015) Development of an improved vaccine evaluation protocol to compare the efficacy of Newcastle Disease vaccines. Biologicals, 43: 136-145.

60. Mohamed, M.H., Abdelaziz, A., Kumar, S., Al-Habib, M. and Mehahed, M.M. (2016) Effect of phylogenetic diversity of velogenic Newcastle disease virus challenge on virus shedding post homologous and heterologous DNA vaccination in chickens. Avian Pathol., 45: 228-234.

61. Sarcheshmei, M., Dadras, H., Mosleh, N. and Mehrabanpour, M.J. (2016) Comparative evaluation of the protective efficacy of different vaccination programs against a virulent field strain of the Newcastle Disease virus in broilers. Braz. J. Poultry Sci., 18: 363-370. 